\title{
Awareness of Mild Bleeding Disorders
}

\author{
a report by \\ Pieter W Kamphuisen
}

Department of Vascular Medicine, Academic Medical Centre, Amsterdam

Inherited bleeding disorders are rare in the general population. Nevertheless, the most common bleeding disorders, haemophilia and von Willebrand disease (VWD), occur in one in 5,000 males and $1 \%$ of the general population. ${ }^{2}$ Since fibrin formation or platelet aggregation in these patients is insufficient, prophylaxis with clotting factor concentrate or desmopressin (DDAVP) prevents severe bleeding complications in situations where optimal haemostasis is crucial, such as surgery. Most patients with either haemophilia or WWD have a relatively mild phenotype, and their disease may well become apparent only after abnormal bleeding postoperatively. In addition, many patients may not be aware of having a bleeding abnormality, since relatives may have the same symptoms Therefore, it is of the utmost importance to identify patients with underlying bleeding disorders before they undergo surgery. In particular, dentists, gynaecologists and paediatricians should investigate patients for potential bleeding abnormalities.

In general, patients with haemophilia can be distinguished from those with VWD by the difference in bleeding symptoms. VWD is caused by a deficiency in or a dysfunction of von Willebrand factor (VWF). WWF is a multimeric protein synthesised in megakaryocytes and endothelial cells. It has two main haemostatic functions. In primary haemostasis at the site of injured vessel walls, it facilitates platelet adhesion to subendothelia structures, such as exposed collagen fibres, and supports platelet aggregation and thrombus formation. As part of secondary haemostasis, VWF acts as a carrier protein for coagulation factor VIII, stabilising and protecting factor VIII pro-coagulant activity. Since VWD has an autosomal dominant inheritance pattern, both men and women can be affected. Individuals with VWD are at increased risk of mucocutaneous bleeding including epistaxis, easy bruising, prolonged bleeding after trivial cuts and dental procedures and reproductive tract bleeding, notably menorrhagia. ${ }^{1,3}$ Haemophilia is an $\mathrm{X}$-linked recessive disorder caused by a partial or complete deficiency of clotting factor VIII (haemophilia A) or factor IX (haemophilia B). Males are typically affected, and bleeding mostly occurs in joints and muscles.

\section{How Can Patients with Mild Bleeding Disorders Be Detected?}

First, one has to be certain that bleeding manifestations are due to an abnormal bleeding diathesis. This is not simple, since the discrimination between patients with mild bleeding disorders and normal subjects is often unclear. In the case of evaluation of a bleeding disorder, patients can be referred because of abnormal bleeding or physicians can actively screen patients pre-operatively for a defect in haemostasis. In the first instance, patients themselves may have suffered from excessive bleeding after trauma or surgery or have spontaneous bleeding or a family history of increased bleeding tendency. One should remember that most of the time, bleeding symptoms are not caused by a specific bleeding disorder. ${ }^{4}$
Table 1: What Questions Should Be Asked to Investigate a Bleeding Diathesis?

Is a pathological bleeding tendency present?

Is it congenital/familial or acquired?

Is it principally a defect of primary haemostasis or of fibrin formation?

Is there a systematic disease causing or exacerbating any bleeding tendency?

In the increased bleeding tendency induced or exacerbated by medication?

For instance, the majority of women with menorrhagia do not have a bleeding disorder, and gynaecological evaluation is required to evaluate other causes of bleeding even in women with a known bleeding disorder. ${ }^{5}$ In a situation where physicians actively screen patients for bleeding disorders, the correlation between coagulation abnormalities as measured in the plasma and the severity of bleeding symptoms is usually very low. ${ }^{6}$ Therefore, the predictive value of an abnormal clotting test alone has hardly any value. Considering these complexities in both clinical and laboratory diagnosis, a systematic approach to the diagnosis of bleeding disorders is a prerequisite.

The clinical approach usually consists of taking a complete medical history and laboratory screening tests. However, there is very little evidence assessing the diagnostic efficacy of clinical history-taking or laboratory screening in order to predict an individual's bleeding risk or to guide clinical management. Nevertheless, it will be worthwhile to identify subjects at risk of excessive bleeding with the use of a simple bleeding history in combination with basic laboratory tests.

\section{Taking Medical History for Bleeding Disorders}

A bleeding history should consist of questions regarding the location and severity of bleeding, familial tendency, co-morbidity and medication such as non-steroidal anti-inflammatory drugs (NSAIDs) or aspirin. Table 1 depicts five important items that should be addressed to investigate the presence of an increased bleeding tendency. ${ }^{4} \mathrm{~A}$ pathological bleeding tendency can consist of large haematomas, menorraghia (preferably since menarche), frequent nose and other mucosal bleeds, easy bruising and excessive bleeding after surgery or trauma. Tonsillectomy or tooth extractions are two examples of frequently occurring forms of surgery that may induce a severe bleeding. A thorough investigation of bleeding symptoms during the whole lifetime should be undertaken, preferably based on objective information. For instance, the physician could ask about the maximum size of ecchymoses or the number of tampons used during menstrual cycles; pictorial charts have also been proposed to assess the amount of menstrual blood losses better. ${ }^{7}$ As a negative bleeding history could also be relevant for the exclusion of a bleeding tendency, all instances of surgery or tooth extraction should also be recorded, even if no haemorrhages occurred. Finally, subjects should also be asked about the type of medical intervention that was required to control bleeding episodes. 
Table 2: Gradation of the Bleeding Score

\begin{tabular}{|c|c|c|c|c|}
\hline \multicolumn{5}{|c|}{ Score } \\
\hline Symptom & 0 & 1 & 2 & 3 \\
\hline Epistaxis & No or trivial & Present & Packing, cauterisation & Blood transfusion or replacement therapy \\
\hline Cutaneous & No or trivial & Petechiae or bruises & Haematomas & Consultation \\
\hline Bleeding from minor wounds & No or trivial & Present (1-5 episodes/year) & Consultation & Surgical haemostasis \\
\hline Oral cavity & No or trivial & Present & Consultation only & Surgical haemostasis/blood transfusion \\
\hline GI bleeding & No or trivial & Present & Consultation only & Surgery/blood transfusion \\
\hline Tooth extraction & No or trivial & Present & Suturing or packing & Blood transfusion \\
\hline Surgery & No or trivial & Present & Suturing or re-surgery & Blood transfusion \\
\hline Menorrhagia & No or trivial & Present & $\begin{array}{l}\text { Consultation, contraceptive } \\
\text { pill use, iron therapy }\end{array}$ & $\begin{array}{l}\text { Blood transfusion, hysterectomy, } \\
\text { dilatation and curretage }\end{array}$ \\
\hline Post-partum haemorrhage & No or trivial & Present, iron therapy & $\begin{array}{l}\text { Blood transfusion, dilatation } \\
\text { and curretage, suturing }\end{array}$ & Hysterectomy \\
\hline Muscle haematomas & No or trivial & Present & Consultation only & Blood transfusion, surgery \\
\hline Haemarthrosis & No or trivial & Present & Consultation only & Blood transfusion, surgery \\
\hline
\end{tabular}

$G /$ = gastrointestinal

The second item is the familial presence of bleeding symptoms. This item has a high discriminatory power (see below). Furthermore, it could point to VWD or a platelet disorder (when a female relative of a male patient is affected) or to haemophilia (only in male subjects). The third item has been discussed above. Joint and muscle bleeds point to a fibrin formation problem, while cutaneous bleedings and menorrhagia could be due to a defect in primary haemostasis. Examples of systemic diseases that can cause an increased bleeding tendency are Ehlers-Danlos and Cushing's syndrome. Finally, the use of medication that may influence haemostasis should be noted. As well as NSAID's and aspirin, other frequently used drugs can impair haemostasis, such as corticosteroids, antibiotics or antiepileptic medication.

By definition, the simultaneous presence of several bleeding symptoms increases the presence of a bleeding disorder. A woman with menorraghia who experienced severe bleeding after tooth extraction will have a higher a priori probability of a bleeding disorder than someone with bleeding after labour. The reporting of bleeding symptoms is often subjective and prone to the specific perception of a patient. Sramek and colleagues investigated the discriminatory power of bleeding symptoms in patients with a bleeding disorder, patients with suspected bleeding abnormality but normal haemostasis and healthy volunteers. ${ }^{8}$ Two important conclusions from this study could be drawn. First, the presence of a bleeding disorder in the family and bleeding after traumatic event were most informative, whereas gum bleeds, epistaxis and blood in the urine did not discriminate between the presence and absence of bleeding abnormalities. The second important observation was the influence of referral. A bleeding history performed in patients referred to a specialised haematology centre was of little value in identifying patients with a bleeding disorder, while in screening situations such as surgery or dentistry these simple questions are very useful.

\section{Bleeding Severity Score}

Since haemorrhagic symptoms are often non-discriminatory, a systematic approach using a standardised questionnaire is recommended. This improves the quality and consistency of data collection, also for research purposes. One of the most promising questionnaires is the one by
Rodeghiero et al. ${ }^{2}$ This bleeding score is generated by summarising the severity of all bleeding symptoms reported by a subject, and is administered and reviewed by a physician. It is graded according to an arbitrary scale from 0 (complete absence of symptoms) to grade 3 if there was some kind of intervention (see Table 2). In the International Multicenter Study (IMS), this bleeding score was evaluated in 215 control subjects. ${ }^{2}$ A bleeding score above three for males and above five for females corresponded to a sensitivity of $64.3 \%$ and a specificity of $99.1 \%$ for the diagnosis of VWD. In the European MCMDM-1VWD study, the score was further evaluated and slightly refined, with a grading from -1 (absence of bleeding despite a haemostatic challenge) to 4 (requiring blood transfusion or surgery to control bleeding). ${ }^{3}$ Retrospectively, 144 patients with type 1 VWD, 273 affected and 295 unaffected family members and 195 controls were included. Only one female control had a bleeding score of $4(0.5 \%)$, while all the remaining control subjects had a lower score. In addition, the likelihood ratio of VWD increased exponentially with each unit increase of the bleeding score: a bleeding score of three points had a likelihood ratio of three, whereas this was 15 in patients with four points. Taken together, this bleeding score seems able to categorise controls as low and high probability of having VWD type 1. This information could then be combined with the VWF level in a patient, which could be used to calculate the final probability of having VWD. ${ }^{9}$ It will be important to validate this bleeding score in other populations, such as children, and in other forms of inherited bleeding disorders. For clinical practice, it would be ideal if the bleeding score correlated with a higher risk of bleeding during surgery.

Recently, a website was launched (www.signsofbleeding.com) that informs physicians about bleeding history and gives background information. Hopefully, with the aid of these bleeding scores and through information over the Internet, subjects who are at risk of bleeding complications during surgery are detected more easily and reliably. In addition, a higher awareness among surgeons, dentists, gynaecologists and general practitioners of potential bleeding abnormalities could reduce bleeding complications.
1. Rodeghiero F, Blood, 1987;69:454-9.

2. Rodeghiero F, Castaman G, Tosetto A, et al., J Thromb Haemost, 2005;3:2619-26.

3. Tosetto A, Rodeghiero F, Castaman G, et al., J Thromb Haemost, 2006:4:766-73.

4. Greaves M, Watson HG, J Thromb Haemost, 2007;5(Suppl. 1):
167-74.

5. Kadir RA, Economides DL, Sabin CA, et al., Lancet, 1998;351:485-9.

6. Chee YL, Crawford JC, Watson HG, Greaves M, Br J Haematol, 2008; 140:496-504.

7. Kadir RA, Economides DL, Sabin CA, et al., Haemophilia,
$1999 \cdot 5: 40-48$

8. Sramek A, Eikenboom JC, Briet E, et al., Arch Intern Med, 1995;155: 1409-15

9. Tosetto A, Castaman G, Rodeghiero F, Blood 2008;111:3998-4003. 